\title{
Exercise Training Attenuates Ovariectomy-Induced Alterations in Skeletal Muscle Remodeling, Apoptotic Signaling, and Atrophy Signaling in Rat Skeletal Muscle
}

\author{
Eun-Jeong Cho ${ }^{1,2}$, Youngju Choi ${ }^{2}$, Jiyeon $\mathrm{Kim}^{2}$, Jun Hyun Bae ${ }^{2}$, Jinkyung Cho ${ }^{3}$, Dong-Ho Park ${ }^{1,2}$, Ju-Hee Kang ${ }^{1,2,4}$, \\ Jin Hwan Yoon ${ }^{5}$, Eunmi Park ${ }^{6}$, Dae Yun Seo ${ }^{7}$, Sukho Lee ${ }^{8}$, Hyo-Bum Kwak ${ }^{1,2}$ \\ ${ }^{1}$ Department of Biomedical Science and Engineering, Inha University, Incheon, Korea \\ ${ }^{2}$ Institute of Sports \& Arts Convergence, Inha University, Incheon, Korea \\ ${ }^{3}$ Department of Sport Science, Korea Institute of Sport Science, Seoul, Korea \\ ${ }^{4}$ Department of Pharmacology, College of Medicine, Inha University, Incheon, Korea \\ ${ }^{5}$ Department of Sport Science, Hannam University, Daejeon, Korea \\ ${ }^{6}$ Department of Food and Nutrition, Hannam University, Daejeon, Korea \\ ${ }^{7}$ Department of Physiology, College of Medicine, Cardiovascular and Metabolic Disease Center, Inje University, Busan, Korea \\ ${ }^{8}$ Department of Counseling, Health, and Kinesiology, Texas A\&M University at San Antonio, TX, USA
}

\begin{abstract}
Purpose: The effects of aerobic exercise training on soleus muscle morphology, mitochondria-mediated apoptotic signaling, and atrophy/hypertrophy signaling in ovariectomized rat skeletal muscle were investigated.

Methods: Female Sprague-Dawley rats were divided into control (CON), ovariectomy (OVX), and ovariectomy plus exercise $(\mathrm{OVX}+\mathrm{EX})$ groups. After ovarian excision, exercise training was performed using a rat treadmill at $20 \mathrm{~m} / \mathrm{min}, 50 \mathrm{~min} / \mathrm{day}, 5$ days/week for 12 weeks. Protein levels of mitochondria-mediated apoptotic signaling and atrophy/hypertrophy signaling in the skeletal muscle (soleus) were examined through western immunoblot analysis.

Results: The number of myocytes and myocyte cross-sectional area (CSA) were increased and the extramyocyte space was decreased in the OVX group compared to those in the CON group. However, aerobic exercise training significantly increased myocyte CSA and decreased extramyocyte space in the OVX+EX group compared to those in the OVX group. The protein levels of proapoptotic signaling and muscle atrophy signaling were significantly increased, whereas the protein levels of muscle hypertrophy signaling were significantly decreased in the OVX group compared to that in the CON group. Aerobic exercise training significantly decreased the protein levels of proapoptotic signaling and increased the protein level of antiapoptotic protein in the OVX+EX group compared to that in the OVX group. Aerobic exercise training significantly increased the protein levels of hypertrophy signaling and decreased protein levels of atrophy signaling in the OVX+EX group compared to those in the OVX group.

Conclusions: Treadmill exercise improved estrogen deficiency-induced impairment in skeletal muscle remodeling, mitochondria-mediated apoptotic signaling, and atrophy/hypertrophy signaling in skeletal muscle.
\end{abstract}

Keywords: Estrogen; Ovariectomy; Sarcopenia; Exercise; Skeletal muscle

- Fund/Grant Support: This work was supported by the Ministry of Education of the Republic of Korea and the National Research Foundation of Korea (NRF-2016R1A2B4014240, NRF-2018R1A2A3074577, NRF-2019S1A5C2A03082727).

- Research Ethics: Animal care and experimental procedures with rats were approved by the Han Nam University Institutional Animal Care and Use Committee (HNU 2018-1).

- Conflict of Interest: No potential conflict of interest relevant to this article was reported.

Corresponding author: Hyo-Bum Kwak (iD https://orcid.org/0000-0003-0451-4554 Department of Biomedical Science and Engineering, Inha University, 100 Inharo, Michuhol-gu, Incheon 22212, Korea

Email: kwakhb@inha.ac.kr

Submitted: September 15, 2021 / Accepted after revision: October 30, 2021
This is an Open Access article distributed under the terms of the Creative Commons Attribution Non-Commercial License (http://creativecommons.org/licenses/by-nc/4.0/) which permits unrestricted non-commercial use, distribution, and reproduction in any medium, provided the original work is properly cited. 


\author{
- HIGHLIGHTS \\ - Estrogen deficiency induces skeletal muscle remodeling. \\ - Exercise reverses estrogen deficiency-induced apoptotic signaling. \\ - Exercise suppresses estrogen deficiency-induced atrophy of skeletal muscles.
}

\section{INTRODUCTION}

The population of women aged 65 years or above is increasing, and their life expectancy has increased to 100 years. Typically, menopause in women occurs between 49 and 52 years of age [1]. Thus, women spend more than one-third of their lives after menopause, which is characterized by low levels of estrogen. Estrogen is a sex steroid hormone that plays a crucial role in human physiology. Therefore, the decline in estrogen levels is related to cardiovascular disease, obesity, type II diabetes [2,3], and osteoporosis [4,5].

In the human body, skeletal muscle is an essential metabolic tissue that accounts for approximately $45 \%$ of the whole-body mass. Rolland et al. [6] demonstrated that muscle mass decreased by $0.6 \%$ per year after menopause. A previous study reported that decreased estrogen levels influenced skeletal muscles and accelerated the pathogenesis of sarcopenia [7], which is the loss of muscle strength, mass, and physical performance [8]. $\mathrm{Akt} / \mathrm{mTOR}$ signaling is a regulator of muscle quality, such as muscle strength and physical performance [9]. Protein kinase $b$ (Akt) regulate protein synthesis, which activates the phosphorylation of eukaryotic translation initiation factor $4 \mathrm{E}$ binding protein 1 (4E-BP1) and ribosomal protein S6 kinase beta-1 (p70S6K) [10], and inactivates forkhead box O (FOXO) transcription factors [11]. A previous study demonstrated that ovariectomy-induced a decrease in muscle Akt phosphorylation, which was related to a decline in skeletal muscle mass [12]. Apoptosis is induced by 3 pathways: (1) sarcoplasmic reticu$\mathrm{lum} / \mathrm{Ca}^{2+}$-driven pathway, (2) cytokine/Fas receptor-driven pathway, and (3) mitochondria-mediated pathway [13]. The mitochondria-mediated pathway is the most important regulator of various diseases related to skeletal muscle $[14,15]$. The Bcl-2 pathway includes antiapoptotic proteins and proapoptotic proteins [13]. The ratio of proapoptotic such as Bcl-2 to antiapoptotic such as Bax proteins manages cellular survival by controlling mitochondrial membrane permeability [13]. Unbalance mitochondrial membrane integrity leads to the release of cytochrome $\mathrm{c}$ from the mitochondria into the cytosol, and it activates caspase- 3 and caspase- 9 , which results in DNA fragmentation and cell death [16]. According to previous studies, estrogen has a crucial role in the inhibition of mitochondriamediated apoptosis in skeletal muscles [17]. However, there are very few studies on the relationship between estrogen and apoptosis.

As a therapeutic intervention, exercise training is well known for its effectiveness in various diseases such as cancer, obesity, and type II diabetes [18-21]. Exercise training is also known to have positive effects on age-related sarcopenia [22,23]. In addition, there are several studies on the effects of exercise on decreased muscle mass induced by estrogen deficiency. Exercise training is considered an important contributor to muscle mass, strength, and quality of life after menopause [24]. Although the importance of exercise training in estrogen deficiency has been established, the effects of exercise training on mitochondriamediated apoptosis and atrophy/hypertrophy signaling in the skeletal muscle have not been elucidated. Therefore, in this study, we hypothesized that moderate aerobic exercise training could ameliorate estrogen deficiency-induced skeletal muscle morphological changes, mitochondria-mediated apoptotic signaling, and atrophy signaling in rat soleus skeletal muscle.

\section{MATERIALS AND METHODS}

\section{Experimental Design}

Female Sprague-Dawley rats were 7-week-old. Rats were divided into 3 groups $(n=6)$ : control $(\mathrm{CON})$, ovariectomy (OVX), and ovariectomy plus exercise (OVX+EX). The sample size was determined using a previous study [25], and the sample size of 6 animals per group was adequate to detect significant results in the current study. Rats were housed under a temperature-controlled room with a 12:12-hour light-dark cycle, and food and water were provided ad libitum. OVX and OVX+EX groups underwent ovarian resection to induce estrogen deficiency at 7 weeks. In the OVX+EX group, exercise training was performed 2 weeks after surgery. After completing treadmill exercise training for 12 weeks, skeletal muscle (soleus) tissues were extracted, and apoptotic signaling and atrophy/hypertrophy signaling were analyzed via western blotting. Histological analysis was used to determine morphological changes after ovariectomy and exercise. 


\section{Animals and Ethical Approval}

All animal experimental procedures were confirmed with the guidelines of the Korean Academy of Medical Science and the regulations stipulated by the National Institutes of Health (NIH). This study was approved by the Han Nam University Institutional Animal Care and Use Committee (HNU 2018-1).

\section{Ovarian Resection}

Rats were anesthetized with ketamine and $2 \%$ xylazine, skin areas were shaved, and exposed skin was cleaned with $70 \%$ alcohol wipe. An incision of approximately $1 \mathrm{~cm}$ was made in the center of the abdomen, and the ovaries were ligated with surgical suture thread. The bilateral ovaries were removed. After ovarian resection, the wound was closed using a synthetic thread, and antibiotics (cefazolin $50 \mathrm{mg} / \mathrm{kg}$ ) were injected into the muscle to prevent infection. Exercise training was conducted 2 weeks after the surgery.

\section{Western Immunoblot Analysis}

The soleus fibers were homogenized using a lysis buffer and centrifuged at 13,000 rpm for 20 minutes. Protein concentration was measured via colorimetric protein assay kit (Bio-Rad, Hercules, CA, USA). Proteins ( $30 \mu \mathrm{g})$ were separated on sodium dodecyl sulfate-polyacrylamide gels and transferred onto a nitrocellulose membrane on ice. The membranes were incubated using 5\% skim milk with Tris-buffered saline which is containing $0.1 \%$ Tween-20 (TBS-T) and then, incubated overnight at $4^{\circ} \mathrm{C}$ with the following primary antibodies: GAPDH, Bcl-2, Bax, cytochrome c (Santa Cruz Biotechnology, Santa Cruz, CA, USA), Akt, p-AKT, mTOR, p-mTOR, p70S6k, p-p70S6k, 4EBP1, p-4E-BP1, FOXO3, MuRF1, and Atrogin-1 (Cell Signaling Technology, Danvers, MS, USA). The membranes were incubated for 1 hour with secondary antibodies: horseradish peroxidase (HRP)-conjugated anti-mouse (Santa Cruz Biotechnology) or goat anti-rabbit IgG-heavy and light chain antibody HRP conjugated. After washing 3 times in TBS-T, an enhanced chemiluminescence detection kit (Thermo Fisher Scientific, Waltham, MA, USA) was used to detect the band. Protein bands was expressed using a ChemiDoc (Bio-Rad). All protein levels were calculated with GAPDH as a ratio.

\section{Hematoxylin and Eosin Staining}

The paraffin block embedded and sectioned soleus muscle was cut to $5-\mu \mathrm{m}$ thickness. Hematoxylin and eosin staining was performed at room temperature to localize the geometry, myo- cyte nuclei, and extramyocyte space. The number of myocytes, myocyte CSA, and extramyocyte space were measured using the Image analysis program (NIH, Bethesda, MD, USA).

\section{RESULTS}

\section{Effects of Ovariectomy and Exercise Training on Morphology in Rat Skeletal Muscle}

The number of myocytes was significantly lower in the OVX group than in the CON group $(\mathrm{P}<0.05)$ (Fig. $1 \mathrm{~A}, \mathrm{~B})$. The myocyte CSA also decreased by $41 \%$ in the OVX group compared to that in the CON group $(\mathrm{P}<0.05)$ (Fig. 1C). In addition, significantly increased extramyocyte space was confirmed in the OVX myofibers compared to that in the CON myofibers $(\mathrm{P}<0.05)$ (Fig. 1D). Conversely, myocyte CSA was significantly increased by $26 \%(\mathrm{P}<0.05)$ (Fig. 1C). Also, the extramyocyte space was significantly decreased by $26 \%$ in the OVX+EX group compared to that in the OVX group $(\mathrm{P}<0.05)$ (Fig. 1D).

\section{Effects of Ovariectomy and Exercise Training on Mitochondria-Mediated Apoptotic Signaling in Rat Skeletal Muscle}

The Bax protein levels in the OVX group were significantly increased by $104 \%$ than those in the CON group $(\mathrm{P}<0.05)$ (Fig. $2 \mathrm{~A})$. In contrast, $\mathrm{Bcl}-2$ protein levels were decreased by $36 \%$ in the OVX group than those in the CON group $(\mathrm{P}<0.05)$ (Fig. $2 \mathrm{~B})$. Taken together, the $\mathrm{Bax} / \mathrm{Bcl}-2$ ratio, which is an early stage of mitochondria-mediated apoptosis, was increased by OVX $(\mathrm{P}<0.05)$ (Fig. 2C). However, Bcl-2 protein levels were increased by $182 \%$ in the OVX+EX group compared with the OVX group $(\mathrm{P}<0.05)$ (Fig. $2 \mathrm{~B})$, suggesting that aerobic exercise training for 12 weeks protected against OVX-induced increase in early mitochondria-mediated apoptotic signaling.

In addition, the protein levels of cytochrome $c$, which is released by the mitochondrial permeability transition pore (mPTP) opening, also showed similar results. The protein levels of cytochrome c were significantly increased by $105 \%$ in the OVX group compared to those in the CON group $(\mathrm{P}<0.05)$ (Fig. 2D). However, the protein levels of cytochrome $\mathrm{c}$ were significantly lower in the OVX+EX group than in the OVX group $(\mathrm{P}<0.05)$ (Fig. 2D).

\section{Effects of Ovariectomy and Exercise Training on Atrophy and Hypertrophy Signaling in Rat Skeletal Muscle}

The protein levels of FOXO3 were significantly increased by $62 \%$ in the OVX group compared to those in the CON group 
$\mathrm{CON}$
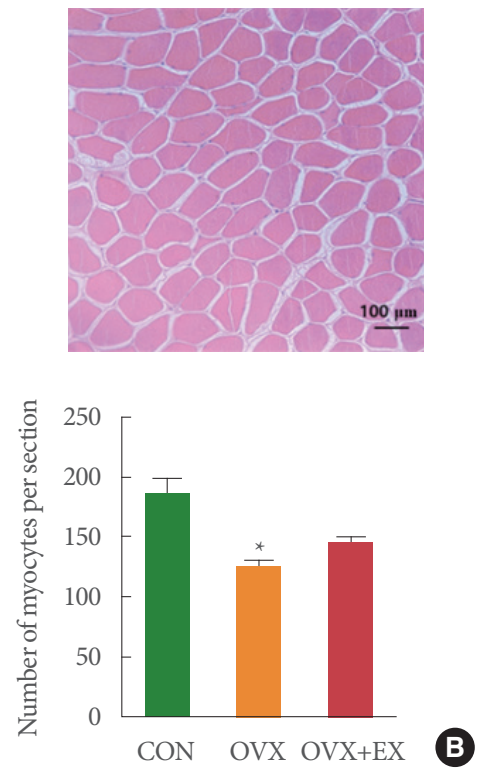

OVX
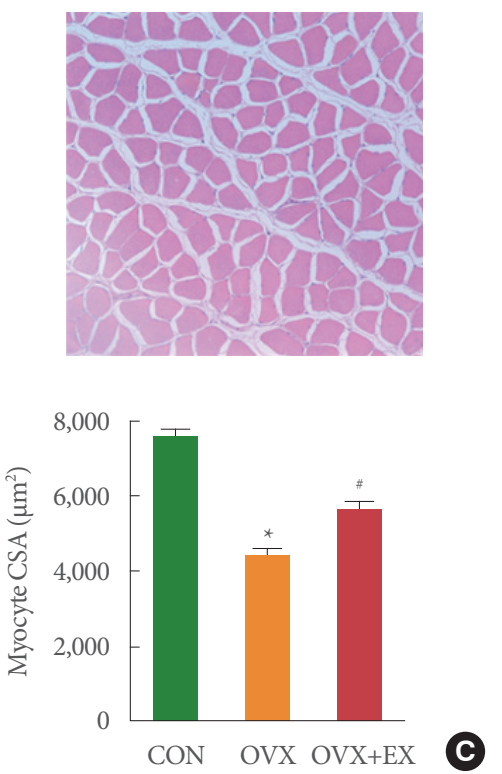

$\mathrm{OVX}+\mathrm{EX}$

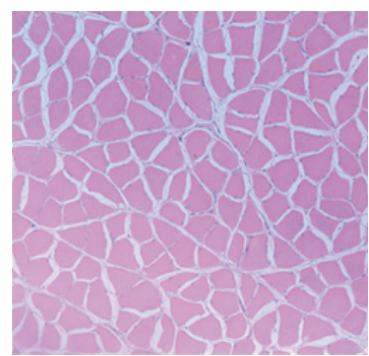

A

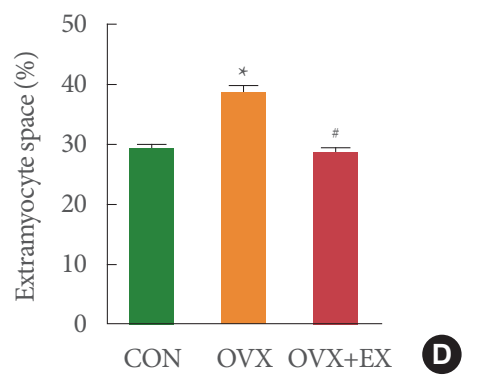

Fig. 1. Effects of ovariectomy and exercise training on morphology. (A) Histological finding. (B) Number of myocytes per section. (C) Myocyte CSA. (D) Extramyocyte space. The data are indicated as the mean \pm standard error of mean. CON, control group; OVX, ovariectomy group; OVX+EX, ovariectomy plus exercise group; CSA, cross-sectional area. ${ }^{\star} \mathrm{P}<0.05$ compared with CON. ${ }^{*} \mathrm{P}<0.05$ compared with OVX.

$(\mathrm{P}<0.05)$ (Fig. 3A). Consistently, MuRF1 and Atrogin-1 protein levels were significantly increased by $40 \%$ and $88 \%$, respectively, in the OVX group compared with the CON group $(\mathrm{P}<0.05)$ (Fig. 3B, C). Conversely, FOXO3, MuRF1, and Atrogin-1 were significantly decreased by $60 \%, 44 \%$, and $42 \%$, respectively, in the OVX+EX group compared with the OVX group $(\mathrm{P}<0.05)$ (Fig. 3A-C), suggesting that 12 weeks of aerobic exercise training attenuated OVX-induced skeletal muscle atrophy.

The protein levels of p-Akt/Akt, p-mTOR/mTOR, p-4E-BP1/ 4E-BP1, and p-p70S6K/p70S6K were significantly decreased by $55 \%, 37 \%, 17 \%$, and $39 \%$, respectively, in the OVX group compared with the CON group $(\mathrm{P}<0.05)($ Fig. 4A-D). However, the protein levels of p-Akt/Akt, p-mTOR/mTOR, and p-p70S6K/ p70S6K were significantly increased by $128 \%, 42 \%$, and $25 \%$, respectively, in the OVX+EX group compared with that in the OVX group $(\mathrm{P}<0.05)($ Fig. 4A-C).

\section{DISCUSSION}

In this study, we demonstrated that aerobic exercise training ameliorated OVX-induced skeletal muscle morphological changes, suggesting that the OVX-induced increase of the connective tissue in the skeletal muscle was reduced and that the decrease in CSA was reversed by aerobic exercise training. Also, we found that aerobic exercise training in OVX rats protected against an OVX-associated increase in mitochondria-mediated apoptotic signaling (e.g., Bax/Bcl-2 ratio and cytochrome c level) in skeletal muscle. In addition, we found that atrophy signaling was decreased, and hypertrophy signaling was increased by aerobic exercise training in ovariectomized rat skeletal muscle. These data support our hypothesis that aerobic exercise training would ameliorate OVX-induced alterations in skeletal muscle morphological changes, mitochondria-mediated apoptotic signaling, and atrophy/hypertrophy signaling in rat skeletal muscle.

Consistent with previous findings [26,27], our study found significant skeletal muscle remodeling, including decreased myocyte number and myocyte CSA, and increased extramyocyte space in the skeletal muscle of ovariectomized rats (Fig. 1), which finally led to the loss of skeletal muscle mass [26]. Many studies have reported the effects of resistance training and hormone replacement therapy as therapeutic interventions for skeletal muscle remodeling [26-28]. However, only a few studies are demonstrating the effect of aerobic exercise, which contributes to increased muscle protein synthesis, on skeletal muscle remodeling in ovariectomized rats. Notably, we observed 

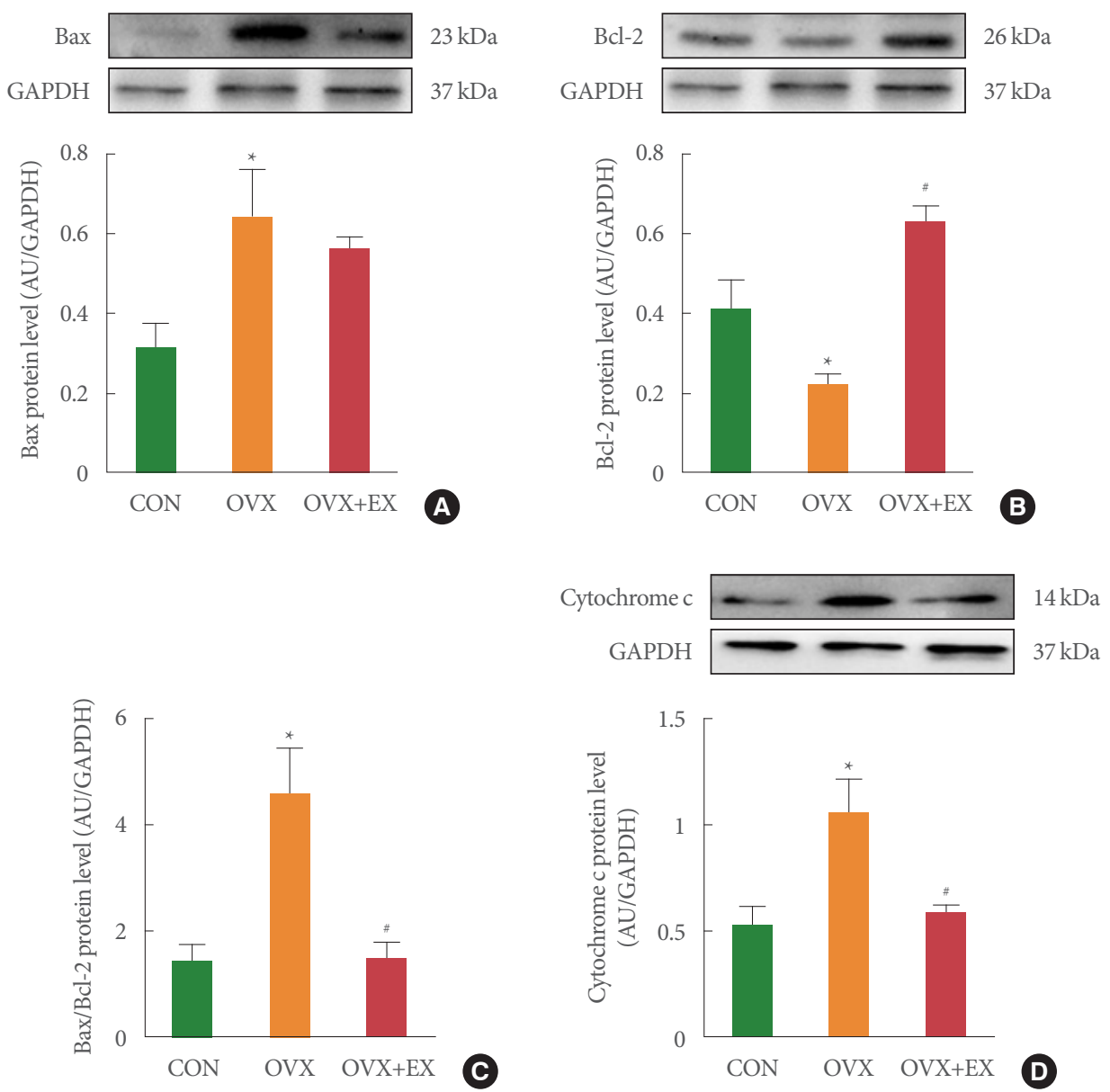

Fig. 2. Effects of ovariectomy and exercise training on mitochondria-mediated apoptotic signaling. (A) Bax. (B) Bcl-2. (C) Bax/Bcl-2 ratio. (D) Cytochrome C. The data are indicated as the mean \pm standard error of mean. CON, control group; OVX, ovariectomy group; OVX+EX, ovariectomy plus exercise group. ${ }^{\star} \mathrm{P}<0.05$ compared with $\mathrm{CON}$. ${ }^{\#} \mathrm{P}<0.05$ compared with $\mathrm{OVX}$.

that aerobic exercise training attenuated estrogen deficiency-induced changes in the myofiber area (CSA) and extramyocyte space. We also observed that aerobic exercise training in OVX rats had no effect on myocyte number, in accordance with previous findings using high-fat diet-induced obesity models [15]. In turn, it seems difficult to expect the effects of aerobic exercise training on skeletal muscle fiber number because skeletal muscles are postmiotic tissues [15]. Therefore, these results suggest that as a therapeutic intervention, aerobic exercise training for 12 weeks exerts beneficial effects on OVX-induced skeletal muscle remodeling.

Mitochondria play an essential role in regulating cell death and survival, also known as mitochondria-mediated apoptosis [29]. We expected that mitochondria-mediated apoptotic signaling could be induced by OVX, and that aerobic exercise training would attenuate mitochondria-mediated apoptotic signaling in the soleus muscles of ovariectomized rats. Indeed, in the current study, the $\mathrm{Bax} / \mathrm{Bcl}-2$ ratio was significantly higher in ovariectomized rat skeletal muscle than in nonovariectomy skeletal muscle (Fig. 2C). This increased $\mathrm{Bax} / \mathrm{Bcl}-2$ ratio by OVX and led to an elevated release of cytochrome $c$ from the mitochondria (Fig. 2D). Moreover, we demonstrated that aerobic exercise training induced the downregulation of cytochrome $\mathrm{c}$ and the $\mathrm{Bax} / \mathrm{Bcl}-2$ ratio in ovariectomized rat skeletal muscle (Fig. 2C, D). Taken together, increased cytochrome c and Bax/ $\mathrm{Bcl}-2$ ratio were markedly attenuated by 12 weeks of aerobic exercise training, suggesting that aerobic exercise training as a therapeutic intervention protects against OVX-induced apoptotic signaling in skeletal muscles. Further studies are needed to confirm that mPTP opening, caspase- 9 , and caspase- 3 cleavage are key regulators of mitochondria-mediated apoptosis, which ultimately lead to DNA fragmentation and cell death [13].

Skeletal muscle mass depends on the dynamic balance between protein degradation and synthesis. Many studies have 

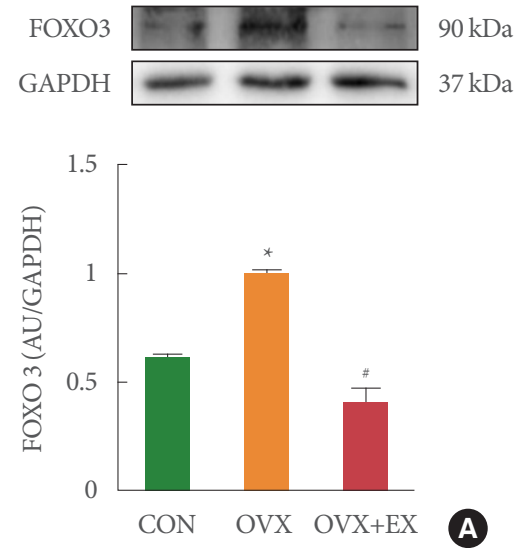
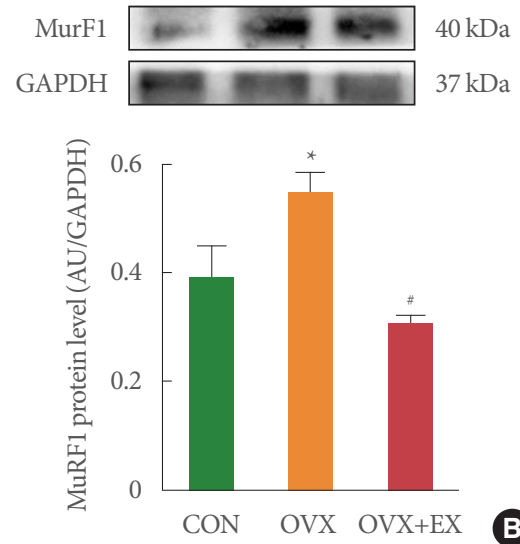
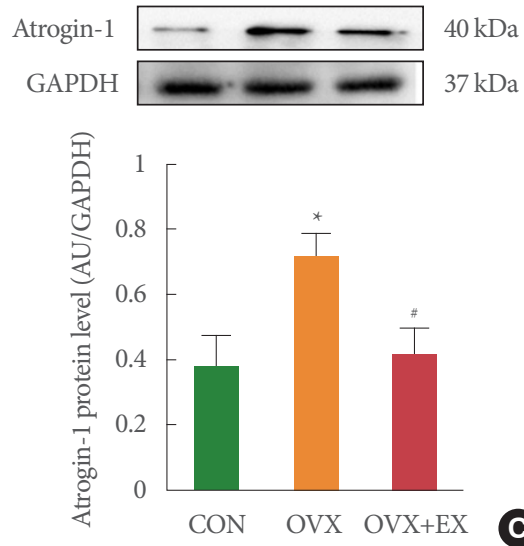

Fig. 3. Effects of ovariectomy and exercise training on atrophy signaling. (A) FOXO3. (B) MurF1. (C) Atrogin-1. The data are indicated as the mean \pm standard error of mean. CON, control group; OVX, ovariectomy group; OVX+EX, ovariectomy plus exercise group; FOXO3, forkhead box O3; MuRF1, muscle ring finger 1; Atrogin-1, muscle specific $\mathrm{f}$ box. ${ }^{*} \mathrm{P}<0.05$ compared with CON. ${ }^{*} \mathrm{P}<0.05$ compared with OVX.
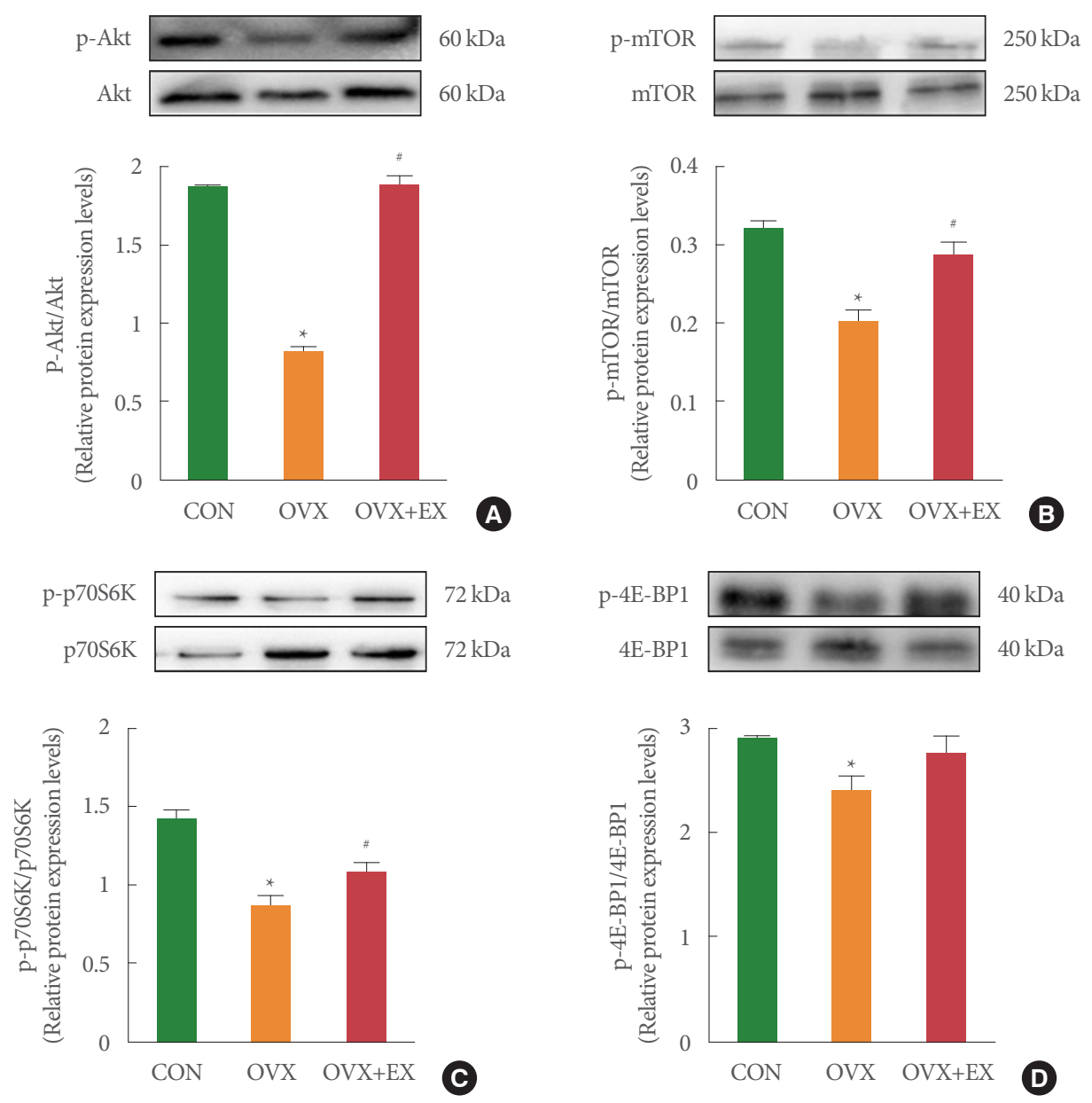

Fig. 4. Effects of ovariectomy and exercise training on hypertrophy signaling. (A) p-Akt/Ak. (B) p-mTOR/mTOR. (C) p-p70S6K/ p70S6K. (D) p-4E-BP1/4E-BP1. The data are indicated as the mean \pm standard error of mean. CON, control group; OVX, ovariectomy group; OVX+EX, ovariectomy plus exercise group; Akt, protein kinase B; mTOR, mammalian target of rapamycin; p70S6K, ribosomal protein $\mathrm{S} 6$ kinase beta-1; 4E-BP1, eukaryotic translation initiation factor $4 \mathrm{E}$ binding protein $1 .{ }^{\star} \mathrm{P}<0.05$ compared with CON. ${ }^{\#} \mathrm{P}<0.05$ compared with OVX. 
clearly confirmed that the Akt/mTOR pathway is important in regulating muscle protein synthesis [30,31], and FOXO3 transcription factor plays a crucial role in muscle atrophy [32], which leads to the expression of MuRF1 and Atrogin-1 [33]. We hypothesized that OVX-induced estrogen deficiency resulted in increased atrophy signaling and decreased hypertrophy signaling in the skeletal muscle. Indeed, the current study demonstrated that muscle atrophy signaling markers such as FOXO3, MuRF1, and Atrogin-1 were increased in the OVX group (Fig. 3). Conversely, in the OVX group, muscle hypertrophy signaling markers such as Akt, mTOR, p70S6K, and 4E-BP1 were decreased (Fig. 4), suggesting that OVX-induced estrogen deficiency results in skeletal muscle atrophy. In addition, we showed that aerobic exercise training attenuated atrophy signaling and recovered hypertrophy signaling in OVX-induced skeletal muscle (Figs. 3, 4). These results suggest that aerobic exercise training protects against OVX-induced skeletal muscle atrophy by activating Akt-mTOR signaling. However, there are only a few studies on aerobic exercise training as a therapeutic intervention for atrophy induced by OVX. Therefore, more studies are needed to verify the effects of aerobic exercise training on the recovery of OVX-induced skeletal muscle atrophy.

Twelve weeks of aerobic exercise training ameliorated OVXinduced changes in skeletal muscle morphology, demonstrating that exercise training ameliorated the OVX-related decrease in myocyte CSA and increase in extramyocyte space. In addition, exercise training in ovariectomized rats protected against OVXinduced increases in mitochondria-mediated apoptotic signaling (e.g., Bax, Bcl-2, cytochrome c) protein levels in the skeletal muscle of ovariectomized rats. Moreover, exercise training attenuated skeletal muscle atrophy signaling (e.g., FOXO3, MuRF1, Atrogin-1) and recovered skeletal muscle hypertrophy signaling (e.g., Akt, mTOR, p70S6K, 4E-BP1) in ovariectomized rats. These findings support the hypothesis that modulation of mitochondria-mediated apoptosis by aerobic exercise training could attenuate OVX-induced skeletal muscle remodeling and thus reduce atrophy of skeletal muscle with estrogen deficiency.

\section{AUTHOR CONTRIBUTION STATEMENT}

- Conceptualization: EJC

- Data curation: $Y C, J K, J H B$

- Formal analysis: $Y C, J K, J H B$

- Funding acquisition: $H B K$

- Methodology: JC, DHP, JHK
- Project administration: $H B K$

- Visualization: JHY, EP, DYS, $S L$

-Writing-original draft: EJC

-Writing-review \& editing: $H B K$

\section{ORCID}

$\begin{array}{ll}\text { Eun-Jeong Cho } & 0000-0003-3683-6523 \\ \text { Youngju Choi } & 0000-0001-8451-3660 \\ \text { Jiyeon Kim } & 0000-0002-4849-8253 \\ \text { Jun Hyun Bae } & 0000-0003-1918-9213 \\ \text { Jinkyung Cho } & 0000-0002-4500-3622 \\ \text { Dong-Ho Park } & 0000-0003-1863-0652 \\ \text { Ju-Hee Kang } & 0000-0001-5235-8993 \\ \text { Jin Hwan Yoon } & 0000-0001-9026-6416 \\ \text { Eunmi Park } & 0000-0002-1911-4652 \\ \text { Dae Yun Seo } & 0000-0002-4377-5792 \\ \text { Sukho Lee } & 0000-0002-8506-2897 \\ \text { Hyo-Bum Kwak } & 0000-0003-0451-4554\end{array}$

\section{REFERENCES}

1. Maltais ML, Desroches J, Dionne IJ. Changes in muscle mass and strength after menopause. J Musculoskelet Neuronal Interact 2009; 9:186-97.

2. Brown LM, Gent L, Davis K, Clegg DJ. Metabolic impact of sex hormones on obesity. Brain Res 2010;1350:77-85.

3. Vogel H, Mirhashemi F, Liehl B, Taugner F, Kluth O, Kluge R, et al. Estrogen deficiency aggravates insulin resistance and induces betacell loss and diabetes in female New Zealand obese mice. Horm Metab Res 2013;45:430-35.

4. Klein-Nulend J, van Oers RF, Bakker AD, Bacabac RG. Bone cell mechanosensitivity, estrogen deficiency, and osteoporosis. J Biomech 2015;48:855-65.

5. Kim KH, Lee HB. Effects of circuit training interventions on bone metabolism markers and bone density of old women with osteopenia. J Exerc Rehabil 2019;15:302-07.

6. Rolland YM, Perry HM 3rd, Patrick P, Banks WA, Morley JE. Loss of appendicular muscle mass and loss of muscle strength in young postmenopausal women. J Gerontol A Biol Sci Med Sci 2007;62: 330-35.

7. Khadilkar SS. Musculoskeletal disorders and menopause. J Obstet Gynaecol India 2019;69:99-103.

8. Cruz-Jentoft AJ, Bahat G, Bauer J, Boirie Y, Bruyère O, Cederholm T, et al. Sarcopenia: revised European consensus on definition and 
diagnosis. Age Ageing 2019;48:601.

9. Shi R, Tian X, Feng Y, Cheng Z, Lu J, Brann DW, et al. Expression of aromatase and synthesis of sex steroid hormones in skeletal muscle following exercise training in ovariectomized rats. Steroids 2019;143:91-6.

10. Bodine SC, Stitt TN, Gonzalez M, Kline WO, Stover GL, Bauerlein $\mathrm{R}$, et al. Akt/mTOR pathway is a crucial regulator of skeletal muscle hypertrophy and can prevent muscle atrophy in vivo. Nat Cell Biol 2001;3:1014-19.

11. Sandri M, Sandri C, Gilbert A, Skurk C, Calabria E, Picard A, et al. Foxo transcription factors induce the atrophy-related ubiquitin ligase atrogin-1 and cause skeletal muscle atrophy. Cell 2004;117: 399-412.

12. Sitnick M, Foley AM, Brown M, Spangenburg EE. Ovariectomy prevents the recovery of atrophied gastrocnemius skeletal muscle mass. J Appl Physiol (1985) 2006;100:286-93.

13. Hengartner MO. The biochemistry of apoptosis. Nature 2000;407: 770-76.

14. Picard M, Ritchie D, Thomas MM, Wright KJ, Hepple RT. Alterations in intrinsic mitochondrial function with aging are fiber typespecific and do not explain differential atrophy between muscles. Aging Cell 2011;10:1047-55.

15. Heo JW, Yoo SZ, No MH, Park DH, Kang JH, Kim TW, et al. Exercise training attenuates obesity-induced skeletal muscle remodeling and mitochondria-mediated apoptosis in the skeletal muscle. Int J Environ Res Public Health 2018;15:2301.

16. Kalkavan H, Green DR. MOMP, cell suicide as a BCL-2 family business. Cell Death Differ 2018;25:46-55.

17. Boland R, Vasconsuelo A, Milanesi L, Ronda AC, de Boland AR. 17Beta-estradiol signaling in skeletal muscle cells and its relationship to apoptosis. Steroids 2008;73:859-63.

18. Seo DY, Heo JW, Ko JR, Kwak HB. Exercise and neuroinflammation in health and disease. Int Neurourol J 2019;23(Suppl 2):S82-92.

19. Park SS, Park HS, Jeong H, Kwak HB, No MH, Heo JW, et al. Treadmill exercise ameliorates chemotherapy-induced muscle weakness and central fatigue by enhancing mitochondrial function and inhibiting apoptosis. Int Neurourol J 2019;23(Suppl 1):S32-9.

20. Ha SY, Han JH, Ko YJ, Sung YH. Ankle exercise with functional electrical stimulation affects spasticity and balance in stroke patients. J Exerc Rehabil 2020;16:496-502.

21. Li S, Kim JY, Sim YJ. Effects of 10-week combined training on lipid metabolic regulatory hormones and metabolic syndrome index according to exercise dose in obese male college students. J Exerc Re- habil 2020;16:101-7.

22. de Freitas MC, de Souza Pereira CG, Batista VC, Rossi FE, Ribeiro AS, Cyrino ES, et al. Effects of linear versus nonperiodized resistance training on isometric force and skeletal muscle mass adaptations in sarcopenic older adults. J Exerc Rehabil 2019;15:148-54.

23. Yoon JR, Ha GC, Kang SJ, Ko KJ. Effects of 12-week resistance exercise and interval training on the skeletal muscle area, physical fitness, and mental health in old women. J Exerc Rehabil 2019;15: 839-47.

24. Elavsky S, McAuley E. Physical activity, symptoms, esteem, and life satisfaction during menopause. Maturitas 2005;52:374-85.

25. Charan J, Kantharia ND. How to calculate sample size in animal studies? J Pharmacol Pharmacother 2013;4:303-06.

26. Gomes RM, Junior MDF, Francisco FA, Moreira VM, de Almeida DL, Saavedra LPJ, et al. Strength training reverses ovariectomy-induced bone loss and improve metabolic parameters in female Wistar rats. Life Sci 2018;213:134-41.

27. Kitajima Y, Ono Y. Estrogens maintain skeletal muscle and satellite cell functions. J Endocrinol 2016;229:267-75.

28. Pighon A, Gutkowska J, Jankowski M, Rabasa-Lhoret R, Lavoie JM. Exercise training in ovariectomized rats stimulates estrogeniclike effects on expression of genes involved in lipid accumulation and subclinical inflammation in liver. Metabolism 2011;60:629-39.

29. Quadrilatero J, Alway SE, Dupont-Versteegden EE. Skeletal muscle apoptotic response to physical activity: potential mechanisms for protection. Appl Physiol Nutr Metab 2011;36:608-17.

30. Latres E, Amini AR, Amini AA, Griffiths J, Martin FJ, Wei Y, et al. Insulin-like growth factor-1 (IGF-1) inversely regulates atrophy-induced genes via the phosphatidylinositol 3-kinase/Akt/mammalian target of rapamycin (PI3K/Akt/mTOR) pathway. J Biol Chem 2005;280:2737-44.

31. Rommel C, Bodine SC, Clarke BA, Rossman R, Nunez L, Stitt TN, et al. Mediation of IGF-1-induced skeletal myotube hypertrophy by PI (3) K/Akt/mTOR and PI (3) K/Akt/GSK3 pathways. Nat Cell Biol 2001;3:1009-13.

32. Clavel S, Siffroi-Fernandez S, Coldefy AS, Boulukos K, Pisani DF, Dérijard B. Regulation of the intracellular localization of Foxo3a by stress-activated protein kinase signaling pathways in skeletal muscle cells. Mol Cell Biol 2010;30:470-80.

33. Mammucari C, Schiaffino S, Sandri M. Downstream of Akt: FoxO3 and mTOR in the regulation of autophagy in skeletal muscle. Autophagy 2008;4:524-26. 